\title{
Les bénéfices sociaux et environnementaux des systèmes agricoles : une analyse ostromienne de trois terrains d'étude en France
}

Social and Environmental Benefits from Farming. An ostromian approach of three case studies in France

Marielle Berriet-Solliec, Christophe Déprés, Colas Chervier, François-Gaël Lataste, Denis Lépicier, Hai Vu Pham et Virginie Piguet

\section{(2) OpenEdition}

\section{Journals}

Édition électronique

URL : http://journals.openedition.org/developpementdurable/17598

DOI : 10.4000/developpementdurable.17598

ISSN : 1772-9971

Éditeur

Association DD\&T

Référence électronique

Marielle Berriet-Solliec, Christophe Déprés, Colas Chervier, François-Gaël Lataste, Denis Lépicier, Hai Vu Pham et Virginie Piguet, «Les bénéfices sociaux et environnementaux des systèmes agricoles: une analyse ostromienne de trois terrains d'étude en France », Développement durable et territoires [En ligne], Vol. 11, n³ | Décembre 2020, mis en ligne le 20 décembre 2020, consulté le 21 janvier 2021. URL : http://journals.openedition.org/developpementdurable/17598; DOI : https://doi.org/10.4000/ developpementdurable.17598

Ce document a été généré automatiquement le 21 janvier 2021.

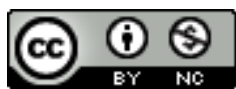

Développement Durable et Territoires est mis à disposition selon les termes de la licence Creative Commons Attribution - Pas d'Utilisation Commerciale 4.0 International. 


\section{Les bénéfices sociaux et environnementaux des systèmes agricoles : une analyse ostromienne de trois terrains d'étude en France}

Social and Environmental Benefits from Farming. An ostromian approach of three case studies in France

Marielle Berriet-Solliec, Christophe Déprés, Colas Chervier, François-Gaël Lataste, Denis Lépicier, Hai Vu Pham et Virginie Piguet

Les auteurs tiennent à remercier les relecteurs anonymes : leurs remarques et commentaires ont été de fructueux aiguillons pour affiner l'analyse et mieux valoriser les résultats de cette recherche.

1 Depuis une dizaine d'années, la réflexion scientifique en économie sur le renouvellement des formes d'accompagnement public des transformations agricoles a largement mobilisé les notions de biens publics (Cooper et al., 2009 ; Lataste et al., 2015) et de services écosystémiques (Braat et de Groot, 2012). La mise en avant de ces deux notions tend à re-légitimer les soutiens publics à l'agriculture dans un contexte d'interpellation des représentants de la société civile sur les effets sociaux et environnementaux positifs et négatifs de ces activités.

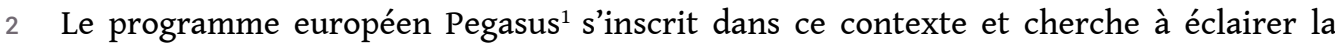
diversité des formes actuelles d'accompagnement public et privé du développement agricole à l'échelle européenne. Cette recherche vise à analyser et comparer un éventail de situations où les pratiques agricoles influent sur la production de biens publics et de services écosystémiques. L'idée initiale est de définir une approche qui permette d'embrasser conjointement ces deux concepts en partant du constat de leur potentielle complémentarité (Dwyer et al., 2018). En effet, des recherches préalables nous ont permis de montrer que l'analyse économique des biens publics reste souvent centrée sur les caractéristiques techniques des biens/services, exprimées en particulier en termes de défaillances de marché (rivalité, exclusion). De telles caractéristiques 
justifient des politiques publiques dites correctrices inspirées de l'économie publique, mais présentent le défaut de ne recouvrir que très partiellement l'éventail des formes actuelles d'accompagnement public du développement agricole en occultant la complexité des situations locales (Lataste et al., 2015 ; Berriet-Solliec, 2016). Quant à l'économie des services écosystémiques, elle aborde davantage les conditions d'émergence de ces services (Froger et al., 2012; Méral et Pesche, 2016) et surtout la manière de les évaluer monétairement (Chervier et al., 2012 ; Chervier et al., 2016b) tout en délaissant, dans une large mesure, la compréhension des mécanismes d'offre de tels services. Ainsi, peu de recherches abordent, dans une vision systémique et appliquée, la dimension collective des processus en jeu visant à encourager la fourniture de bénéfices sociaux et environnementaux associés aux activités productives.

Dès lors, en proposant d'utiliser le terme plus générique de "bénéfices sociaux et environnementaux $^{2}$ » (BSE), notre recherche vise à caractériser, à l'échelle locale, les systèmes dans lesquels les activités agricoles sont conduites, et à analyser les facteurs favorisant ou limitant la production de ces bénéfices, qu'ils soient fournis à une échelle locale ou plus globale, appropriés de manière individuelle et/ou collective, faisant l'objet ou non de rivalité. Il ne s'agit pas dans cet article d'analyser la valeur des biens ni les dispositifs qui permettent leur évaluation, notions pourtant centrales chez Ostrom (Allaire, 2013). Nos travaux portent sur une première phase de la démarche ostromienne qui consiste en la mise en lumière et la reconnaissance de ces biens (Dwyer et al., 2018). Cette démarche a été appliquée en France sur trois terrains retenus au regard de la diversité de leur contexte agricole et institutionnel, et de l'intérêt des problématiques soulevées: préservation des paysages dans une zone agricole intermédiaire (Pays de Langres), qualité et disponibilité des eaux souterraines dans une région d'élevage (impluvium de Volvic), durabilité des paysages dans un système méditerranéen agropastoral (Parc national des Cévennes). Il ne s'agit pas de comparaison, mais de mise en regard de trois études de cas répondant ainsi au besoin de multiplier les analyses empiriques approfondies (Allaire, 2013).

4 L'article est structuré en quatre parties : après ces propos liminaires, nous présentons, en première partie, notre cadre d'analyse et notre démarche méthodologique. La partie 2 est dédiée à la présentation des résultats, et la partie 3 à une conclusion et à des enseignements relatifs à l'action collective dans les espaces ruraux.

\section{Cadre analytique et démarche méthodologique}

\subsection{Un cadre d'analyse mobilisant les travaux d'Elinor Ostrom}

Dans la mesure où ils décrivent des situations de ressources communes, en explorent les mécanismes de production ainsi que les actions collectives permettant de gérer et préserver les bénéfices sociaux et environnementaux fournis (Allaire, 2013; Allaire, 2019), les travaux d'Ostrom sur la gestion des communs $(1990,2005,2007)$ constituent le cadre d'analyse principal de nos recherches. Depuis 2009 et l'obtention conjointe du prix Nobel d'économie par Ostrom et Williamson, une impulsion nouvelle a ainsi été donnée aux travaux institutionnalistes portant sur la gouvernance. Cette reconnaissance académique a encouragé la poursuite des travaux initiaux, en particulier, avec la reprise du "social and ecological system » (SES) ou « système socioécologique» (SSE en français) par McGinnis et Ostrom (2014). Cet outil conceptuel, 
précédemment conçu par Berkes et al. (2002), permet de prendre en compte l'interconnexion des objets de la nature avec les activités humaines.

L'approche d'Ostrom envisage les "communs" sous l'angle d'une situation où des utilisateurs conjoints d'une ressource commune sont rivaux et interdépendants, ce qui la différencie du cas des biens publics. La rivalité menace ainsi la pérennité de la ressource en cas de surutilisation. L'enjeu apparaît pour Ostrom de comprendre de quelle manière "un groupe d'appropriateurs ${ }^{3}$ de ressources complexes et incertaines parviennent à s'organiser eux-mêmes pour résoudre efficacement des problèmes de mise en place d'institutions, d'engagement et de surveillance [des comportements individuels rationnels et potentiellement opportunistes ou de free-riders] » (Ostrom, 1990 : 43-44).

7 Le cadre SSE se présente dès lors comme une matrice interprétative qui part d'une problématique caractérisée par les auteurs (McGinnis et Ostrom, 2014) de "situationaction » (SA) et qui propose de mettre cette SA en interconnexion avec quatre variables principales présentées dans la figure 1 ci-dessous.

Figure 1. Cadre conceptuel du Système socio-écologique (SSE)

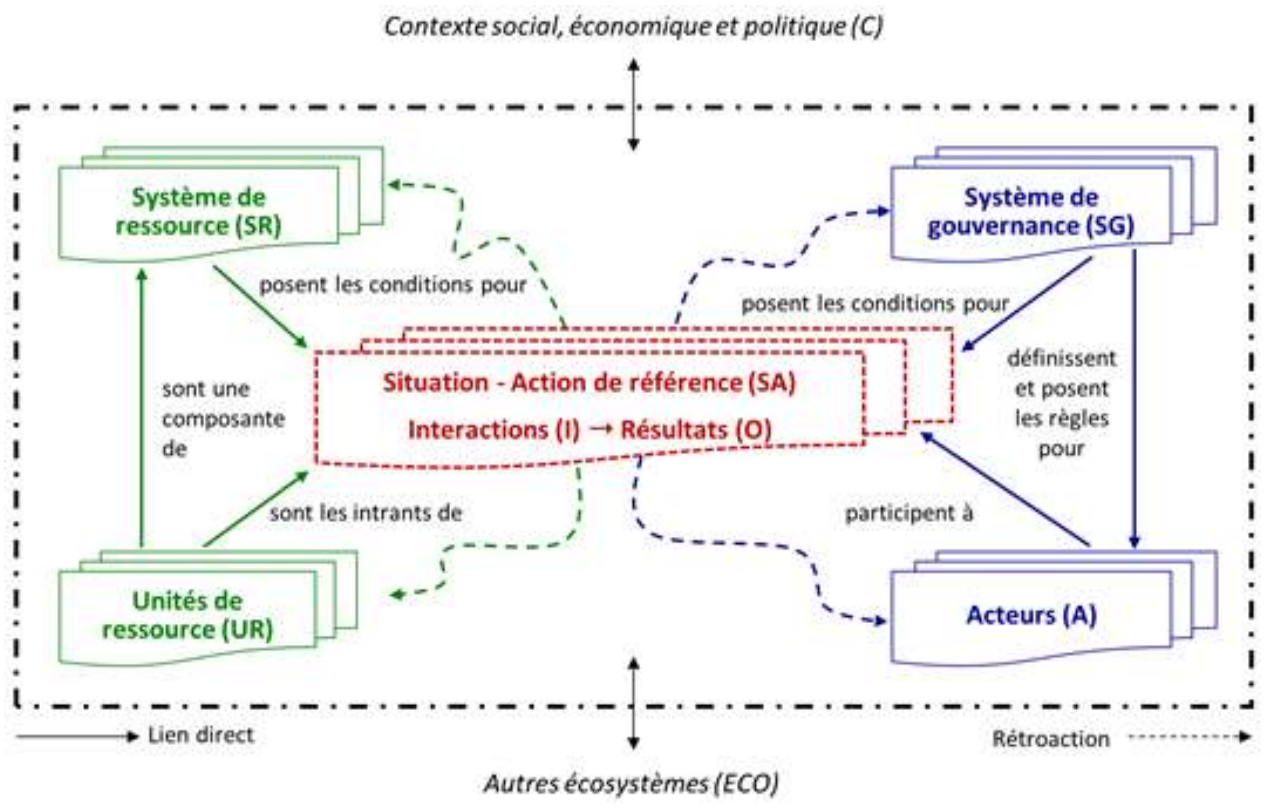

Source : version révisée du cadre SSE issue et traduite de McGinnis et Ostrom (2014)

Dans cette conceptualisation, le terme de «ressource commune » désigne d'abord un système de ressource (assimilable à un stock) suffisamment important pour qu'il soit coûteux (mais pas impossible) d'exclure ses utilisateurs potentiels de l'accès aux bénéfices liés à son utilisation (Ostrom, 1990: 44). Le système de ressource (SR) est distinct (tout en étant interdépendant) des unités de ressource (UR), assimilables à un flux et produites par le système. Le SR dépasse toutefois l'idée de simple stock émettant des flux d'UR appropriables et peut être davantage considéré comme une infrastructure fournissant différents services (une bibliothèque) dont l'homme bénéficie via les UR (les livres). Allaire (2013) illustre la situation de la manière suivante : une bibliothèque n'est pas juste un stock de livres, mais aussi le bâtiment, le personnel, le service d'enregistrement, etc. Bref, tout ce qui permet au service de prêt de fonctionner. Pour gérer cette "infrastructure ", il faut avoir un système fixant les règles de fonctionnement. C'est le rôle du système de gouvernance, qui englobe tous les 
acteurs concernés par le(s) service(s) rendu(s). Ensuite, les acteurs (A) participent à un système de gouvernance $(\mathrm{SG})$ qui définit un ensemble d'interactions techniques, marchandes ou non marchandes, individuelles ou collectives qu'ils entretiennent avec le système de ressource. Enfin, ces quatre variables principales sont à considérer dans un contexte social, économique et politique englobant (C) et en lien avec d'autres systèmes écologiques (ECO), ces deux variables pouvant être rapprochées de la notion d'« atmosphère » dans la métaphore de Marshall (1890).

9 Les liens entre les quatre variables du modèle (UR, SR, A et SG) sont multiples. Nous y trouvons le lien direct entre UR et A, qui est classiquement étudié par la science économique. Les unités représentant une valeur (économique) sont appropriées par les acteurs qui les prélèvent pour les échanger et/ou les transformer sur le marché. Mais le modèle SSE contient également les liens divers entre le SR et le SG. Une des difficultés est que le contour de ces objets n'est pas toujours fixe et/ou clairement identifiable. Le SG résulte d'un ensemble de sous-systèmes encastrés: le droit, les normes, les coutumes et les arrangements locaux. Chaque acteur peut agir sur tout ou partie de cet ensemble ou simplement sur une partie du système de ressource lui-même (Allaire, 2013). C'est, par exemple, le cas (dans la situation de Volvic détaillée ci-après) du comité de gestion de la qualité de l'eau qui se distingue de celui agissant sur la quantité d'eau autorisée à être prélevée annuellement. Quant au SR, il ne fournit pas un seul et unique service (correspondant à l'UR). Les services de l'agriculture ne se limitent pas à la seule fourniture de biens alimentaires, mais incluent aussi les activités d'entretien de chemin permettant l'accès pour les randonneurs, le paysage pour les touristes, etc.

10 C'est ici que la notion de BSE que nous proposons trouve sa place et sa pertinence dans le modèle SSE. En effet, une des idées qui sous-tend le travail d'Ostrom est que les services rendus par le SR ne sont pas tous des UR divisibles dont on peut s'approprier de façon exclusive et privative les bénéfices. Les services écologiques, culturels ou les bienfaits d'un système agricole (tel que le paysage ou le maintien d'une identité locale) peuvent bénéficier conjointement à des individus multiples et potentiellement rivaux. Mais l'existence de ces BSE (dont une part est collective) n'empêche pas que certaines UR continuent d'être extraites et appropriées comme des biens privés dont la gestion repose sur des mécanismes de marché. Il est d'ailleurs assez fréquent que le SR relève d'un régime de propriété commune alors que les UR sont appropriées de manière privative, comme c'est le cas dans les systèmes d'irrigation agricole, exemple maintes fois cité dans les travaux sur les SSE (Mc Ginnis et Ostrom, 2014). Toutefois, le niveau de fourniture de BSE serait menacé si le SR était surexploité sur certains aspects et que le système de gouvernance considérait uniquement les bénéfices privés associés aux activités productives. Le SG peut alors être compris comme l'ensemble des arrangements institutionnels (règles de droits, d'allocation, d'exclusion, de sanction) adaptés à chaque situation-action (Allaire, 2019) afin de maintenir des liens suffisamment fonctionnels entre les composantes du système pour qu'il garantisse le niveau de fourniture des bénéfices sociaux et environnementaux et leur partage entre les acteurs du système. Cela suppose de prendre en compte le SR en totalité - ne pas isoler les composantes du système les unes des autres - et dans toute sa complexité considérer la dynamique propre du système ("ecological rules») qui n'est pas systématiquement connue par les acteurs de manière complète et parfaite.

11 La description du SSE nous permet de poser les concepts clés de la pensée d'Ostrom. Le cadre SSE éclaire le lien entre le commun et les unités de ressource ainsi que 
l'importance du système de gouvernance. Jusqu'à présent, le cadre SSE a été surtout appliqué à des cas de ressources communes relativement bien délimitées, encore appelées «common pool resources» et traditionnellement étudiées par Ostrom dans le cas des pêcheries (Basurto et al., 2013) ou par Cox sur les systèmes d'irrigation (2014). La démarche a certes été plus largement mobilisée pour traiter d'objets comme les sols (Dominati et al., 2010), les écosystèmes des deltas (Brondizio et al., 2016) ou encore le bien-être humain et les compromis (Howe et al., 2014). Mais le cas de ressources territoriales, comme le paysage ou la gestion qualitative de l'eau (Barataud et al., 2018), semble à ce jour encore peu étudié sous l'angle des SSE. La possible application de ce modèle à la gestion de tels systèmes complexes (multi-acteurs, multi-ressources, multiéchelles spatiales) demeure un défi et une question ouverte dont nous nous saisissons dans cet article.

Le présent article restitue donc la manière dont ce cadre d'analyse permet de rendre compte, à partir de trois situations, de la nature des bénéfices environnementaux et sociaux de l'agriculture, et des facteurs pouvant expliquer leur production autour de trois dimensions d'analyse :

- parvenir à une compréhension détaillée des systèmes agricoles locaux et identifier les bénéfices sociaux et environnementaux (BSE) qui en découlent;

- identifier comment ces systèmes agricoles et BSE sont influencés, orientés par les choix productifs, injonctions politiques, signaux du marché et initiatives locales;

-identifier les facteurs clés de motivation, institutionnels et socio-économiques qui permettent le développement des BSE.

\subsection{Méthodologie}

13 En mobilisant une telle approche située et systémique, il est illusoire de rechercher un quelconque modèle universel, tant apparaissent d'emblée multiples les déterminants qui spécifient une situation locale vis-à-vis d'une autre (Benko et al., 1996). Au contraire, ce qui semble essentiel avec cette visée compréhensive, c'est la démarche et les mécanismes qui visent à expliciter les logiques d'organisation dans un contexte géographique et historique précis.

14 Le programme Pegasus recherche ainsi des pistes de solution pour favoriser la production de bénéfices environnementaux et sociaux à partir de l'agriculture dans la diversité des initiatives conduites à l'échelle locale. La contribution de ce programme sera dès lors d'expliciter les logiques du SSE dans un contexte historique et géographique qu'il s'agira de définir au préalable. Pour éviter l'écueil de réduire le SSE au territoire strictement local, nous intégrerons dans le raisonnement les influences extra-territoriales : rôle de l'Unesco (Organisation des Nations unies pour l'éducation, la science et la culture), tensions sur les marchés du lait, Politique agricole commune (PAC), stratégie de la multinationale Danone...

15 Cet article présente les résultats des trois études menées en France (figure 2) et portant sur trois types de situation-action vis-à-vis de l'agriculture (Berriet-Solliec et al., 2016) ${ }^{4}$ : la préservation de paysages originaux en mosaïque en zone agricole intermédiaire dans le Pays de Langres, la préservation du paysage, de la qualité et de la quantité des eaux souterraines dans le périmètre de l'impluvium de Volvic situé en zone d'élevage, enfin la conservation de paysages méditerranéens agropastoraux ancestraux dans le Parc national des Cévennes, seul parc national français métropolitain habité en permanence. 
Dans les trois cas, les paysages peuvent être considérés comme "remarquables » au regard de critères géologiques, géographiques et d'occupation du sol de ces territoires, mais aussi de la perception tant par les habitants que par les acteurs institutionnels, notamment ceux du secteur du tourisme. Pour les Cévennes et la chaîne des Puys faille de Limagne dans lequel est inclus l'impluvium de Volvic, ce caractère remarquable a été reconnu par leur inscription au patrimoine mondial de l'Unesco respectivement en 2011 et 2018.

Figure 2. Localisation des trois études de cas

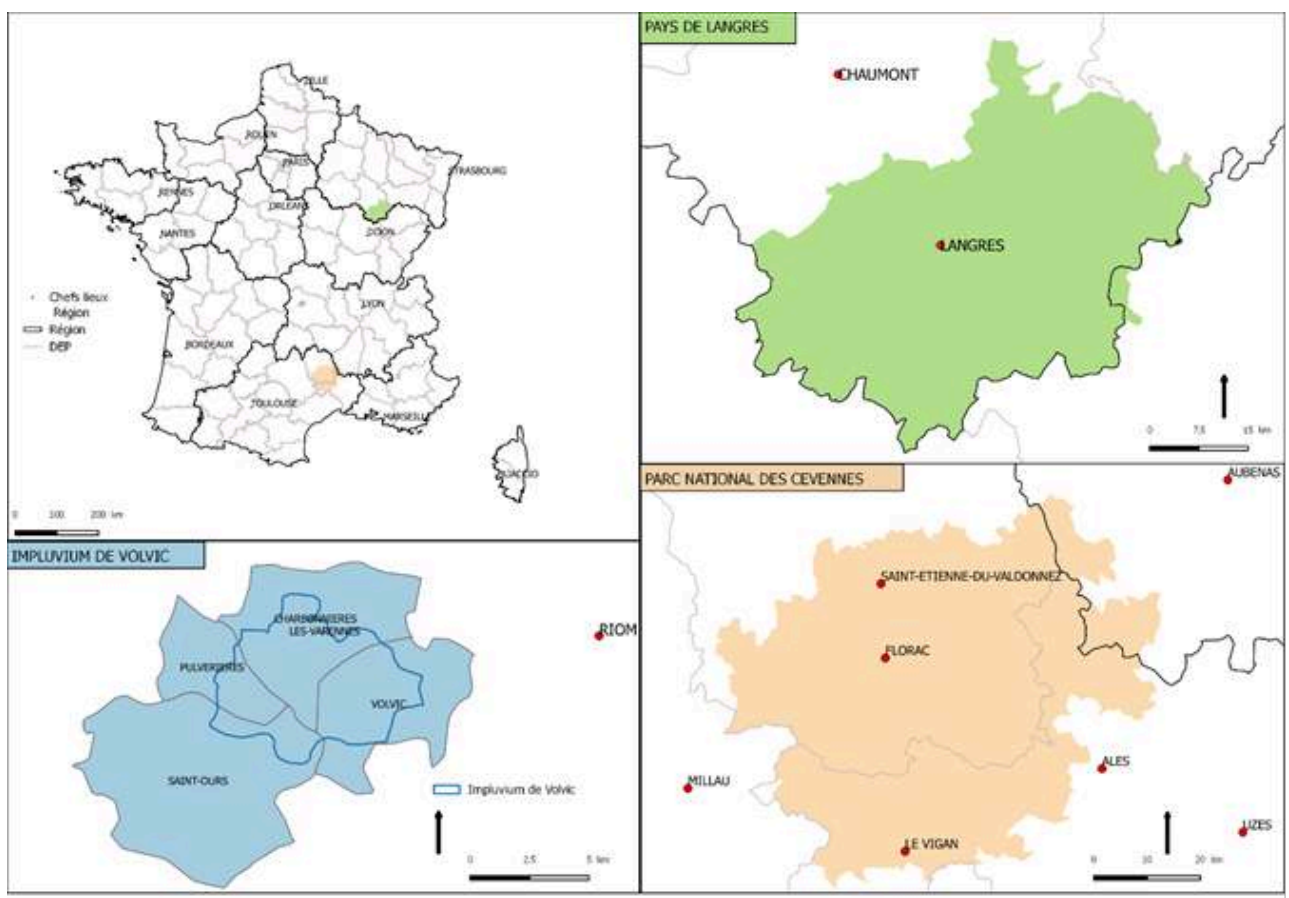

Source : IGN Geofla 2015, réalisation Cesaer 2019. l'agriculture ont permis de recueillir le point de vue de 15 acteurs agricoles (conseillers 
de chambre d'agriculture et association de développement agricole, conservatoire des espaces naturels, agriculteur, élu local).

À Volvic (Chervier et al., 2016a), des entretiens et un atelier participatif ont touché une quinzaine d'acteurs du territoire de l'impluvium: élus des communes concernées, agriculteurs (éleveurs), représentants de Danone (firme exploitant localement l'eau de Volvic), de la Ligue de protection des oiseaux (LPO), de l'association locale de protection de l'impluvium, experts et chercheurs.

Dans le Parc national des Cévennes (Lataste et Piguet, 2016), sept entretiens ont été réalisés avec les principaux acteurs de la gouvernance locale: le Parc national, l'Entente interdépartementale des Causses et Cévennes, deux associations paysannes (Terre et humanisme et la marque départementale collective "De Lozère »), l'association des professionnels du tourisme (Cévennes écotourisme), la structure locale d'enseignement supérieur et de recherche (SupAgro Florac), la direction départementale du territoire (service déconcentré de l'État).

Les entretiens et ateliers ont été guidés par la présentation de la démarche d'Ostrom avec les différentes composantes du SSE (figure 1). Afin d'établir le SSE de chaque terrain d'étude, des informations ont été collectées sur chaque composante grâce à une grille de questionnement commune (tableau 1). Cette grille était composée :

- en colonnes, des composantes du diagramme SSE c'est-à-dire les unités de ressource (UR), le système de ressource (SR), la situation-action de référence (SA), les acteurs $(A)$, le système de gouvernance (SG), les autres écosystèmes (ECO), le contexte social, économique et politique (C) ;

- en lignes, de neuf questions permettant d'identifier comment les différents éléments du SSE interagissent avec la production ou la gestion des trois principaux BSE étudiés, à savoir la gestion d'un paysage en mosaïque à Langres, la qualité et la quantité d'eau à Volvic, la préservation de paysages agropastoraux ancestraux dans le Parc des Cévennes.

Tableau 1. Grille de questionnement utilisée pour les entretiens et ateliers

\begin{tabular}{|l|l|l|l|l|l|l|l|}
\hline & UR & SR & SA & A & SG & ECO & C \\
\hline $\begin{array}{l}\text { Quelles sont les principales caractéristiques des bénéfices sociaux et } \\
\text { environnementaux (BSE) des activités agricoles? }\end{array}$ & X & & & X & X \\
\hline $\begin{array}{l}\text { Quels sont les principaux acteurs impliqués dans la production, la } \\
\text { gestion, voire la destruction de ces BSE? }\end{array}$ & & X & X & X & & \\
\hline $\begin{array}{l}\text { Votre organisation/structure participe-t-elle à la production ou à la } \\
\text { régulation de ces BSE? Si oui, selon quels mécanismes? }\end{array}$ & X & X & X & X & & \\
\hline $\begin{array}{l}\text { Quels sont les principaux facteurs positifs et négatifs qui influent sur } \\
\text { la production des BSE? Selon quels mécanismes? }\end{array}$ & & X & X & X & X & X \\
\hline $\begin{array}{l}\text { Quels sont les principaux bénéficiaires des BSE? Selon quels } \\
\text { mécanismes? }\end{array}$ & & X & X & X & & X \\
\hline $\begin{array}{l}\text { Quelles sont les initiatives publiques ou privées qui contribuent à } \\
\text { gérer ces BSE? Selon quels mécanismes? }\end{array}$ & & X & X & X & & \\
\hline $\begin{array}{l}\text { Y a-t-il des effets pervers ou non attendus de ces BSE? Si oui, quels } \\
\text { sont les mécanismes qui les induisent? }\end{array}$ & X & X & X & X & X & X & X \\
\hline
\end{tabular}




\begin{tabular}{|l|l|l|l|l|l|l|l|l|}
\hline $\begin{array}{l}\text { Existe-t-il des initiatives locales qui interagissent négativement ou } \\
\text { positivement dans la production des BSE? }\end{array}$ & X & X & X & X & X & X & \\
\hline $\begin{array}{l}\text { Quels sont les principaux acteurs qui participent de la gouvernance } \\
\text { mise en place pour la production des BSE? }\end{array}$ & & & X & X & & X \\
\hline
\end{tabular}

Source : traduit et adapté de Berriet-Solliec et al. (2018)

Les informations qualitatives tirées des entretiens ont été complétées par des données statistiques de l'institut national de la statistique (Insee) et du ministère de la Transition écologique et solidaire. Selon la disponibilité des sources en format SIG ou à l'échelle communale, des traitements géomatiques ou des agrégations des données des communes situées dans le périmètre de l'étude de cas ont été réalisés afin de caractériser chaque zone ${ }^{5}$.

Enfin, un troisième temps a consisté en l'organisation d'un séminaire national en octobre 2017 à Dijon qui a réuni 25 participants: représentants institutionnels (ministères en charge de l'Agriculture et de l'Écologie), agricoles et forestiers (syndicats, chambre d'agriculture), environnementaux (parc naturel régional, agence de l'eau) et chercheurs. L'objectif était double : (i) confronter les premiers résultats d'analyse issus des enquêtes de terrain aux acteurs locaux et aux représentants régionaux ou nationaux; (ii) identifier les enseignements pouvant être tirés de la mise en regard des trois terrains.

\section{Gouvernance territoriale et bénéfices sociaux et environnementaux}

\subsection{Caractérisation des trois territoires d'étude}

Les trois terrains d'étude ont été retenus pour leur diversité géographique (zone continentale et méridionale) et institutionnelle (un Pays, un Parc, un impluvium) et pour le caractère original des enjeux soulevés par les situations-actions (conservation d'un paysage en mosaïque, maintien de la quantité et de la qualité de l'eau, entretien d'un paysage agropastoral ancestral).

Dans le premier cas d'étude, le territoire du Pays de Langres, organisé autour de la ville de Langres dans le sud de la Haute-Marne, est un bassin de vie de 172 communes comptant 45000 habitants (tableau 2) et qui couvre $2275 \mathrm{~km}^{2}$ (tableau 3). La géographie de plateaux calcaires et vallées profondes forge un paysage remarquable composé d'une mosaïque d'espaces ouverts sur les plateaux, et de forêts et prairies dans les vallées contribuant à l'identité locale. Il correspond à un territoire de développement local structuré en Pays, puis Pôle d'équilibre territorial et rural (PETR), créé en 2002 en application de la loi Voynet de 1999 relative au développement durable des territoires. Il se définit par son caractère rural de faible densité, faiblement attractif (en perte de population) et distant des grandes métropoles régionales. Le Pays est le support d'un programme de développement rural Leader (Liaison entre les actions de développement de l'économie rurale) depuis 2000.

L'agriculture et la forêt occupent une forte proportion du territoire (respectivement $64 \%$ et $34 \%$, cf. tableau 3) avec un poids économique de l'agriculture supérieur à la 
moyenne nationale (plus de $7 \%$ en 2014, pour $2,7 \%$ en moyenne nationale). L'agriculture est orientée vers les grandes cultures au potentiel caractéristique des zones dites intermédiaires ${ }^{6}$ valorisées par des rotations courtes (céréales et oléoprotéagineux). Ces productions végétales couvrent la moitié des terres arables, mais dégagent $77 \%$ de la valeur de la production agricole (recensement agricole de 2010). Leurs filières de collecte et de transformation sont gérées dans de grands groupes coopératifs ou privés à destination des marchés nationaux et internationaux de matière première agricole faiblement transformée. L'autre moitié des terres cultivables est occupée en prairies dans les vallées et dans les zones plus bocagères du Bassigny, support d'élevages laitiers en déclin sur le long terme et d'élevages allaitants de grande taille et modernisés. Une tradition de transformation fromagère ancienne existe sur le territoire. Elle a conduit à la constitution, en 1981, d'une interprofession du fromage de Langres $^{7}$ qui regroupe 19 producteurs. Ce fromage est certifié par une Appellation d'origine contrôlée (AOC) depuis 1991 et par une Appellation d'origine protégée (AOP) depuis $2009^{8}$.

Le second cas est celui de Volvic et porte sur un territoire plus restreint correspondant à l'aire d'alimentation des sources des eaux de Volvic. Il est situé à l'extrémité nord de la chaîne des Puys - faille de Limagne, inscrite au patrimoine mondial de l'Unesco. L'impluvium de Volvic, d'une surface de $38 \mathrm{~km}^{2}$, s'étend sur les territoires des quatre communes de Volvic, Charbonnières-les-Varennes, Pulvérières et Saint-Ours dans le département du Puy-de-Dôme (figure 2). Il se trouve dans une zone de début de montagne entre 500 et 1000 mètres d'altitude. La population des communes concernées par l'impluvium est d'environ 8000 habitants. La densité communale, faible, varie entre $30 \mathrm{hab} . / \mathrm{km}^{2}$ et $160 \mathrm{hab} . / \mathrm{km}^{2}$ (à Volvic). À $15 \mathrm{~km}$ seulement de Clermont-Ferrand, la plus grande agglomération de la région, l'impluvium est connu comme un des sites de production d'eau de plus grande qualité en France. Rappelons que la France compte 6500 aires d'alimentation de captage, dont 1300 environ possèdent une superficie supérieure à $10 \mathrm{~km}^{2}$ comme l'aquifère de Volvic (Petit, 2009). Sur l'impluvium de Volvic, une grande partie du sol appartient à des propriétaires privés. Il s'agit d'espaces de forêt et de terres agricoles composées essentiellement de prairies naturelles de pâturage. En 2012, la forêt représente ainsi $56 \%$ de la zone, soit $21,25 \mathrm{~km}^{2}$, les territoires agricoles et les milieux semi-naturels $38 \%$ soit $18,58 \mathrm{~km}^{2}$ et les espaces artificialisés (routes, constructions) demeurent relativement limités avec un peu moins de $6 \%$ des surfaces. Par ailleurs, le territoire héberge des espèces protégées (milan royal et chauve-souris).

Enfin, le troisième cas retenu, le Parc national des Cévennes, correspond à un système agropastoral méditerranéen d'utilisation des terres avec des caractéristiques originales reconnues par l'Unesco, notamment les rapports ancestraux entretenus entre l'homme et la nature depuis le Moyen Âge. Situé dans le sud du centre de la France, le Parc national des Cévennes a été créé en 1970 pour répondre à la fois au départ des populations autochtones et à l'arrivée d'une population néorurale fuyant la ville, en quête de qualité de vie et de retour à la nature.

Ce parc national est un territoire d'un peu moins de $3800 \mathrm{~km}^{2}$ traversé par trois entités territoriales : les vallées des Cévennes, les hautes terres des Causses et le mont Lozère. Les entretiens ont permis de distinguer clairement ces trois sous-systèmes qui correspondent aux petites régions agricoles définies par la statistique agricole. Sur le plan méthodologique, pour mener la comparaison avec les deux autres cas, nous 
considérerons l'unité du Parc national des Cévennes comme un territoire avec sa propre identité, mais les analyses ont été approfondies dans les trois zones Causses/ Cévennes/mont Lozère où les conditions sociales et naturelles (sols, végétation, relief...) influent de façon spécifique sur le fonctionnement du SSE.

Tableau 2. Caractéristiques démographiques

\begin{tabular}{|l|l|l|l|l|}
\hline & Langres & Volvic & Cévennes & France \\
\hline Population 2014 (N) & 45661 & 8115 & 75267 & 64027958 \\
\hline Densité 2014 (hab./km²) & 20 & 62 & 20 & 117 \\
\hline Taux de variation annuel 1990-2014 de la population (\%) & $-0,4$ & 0,84 & 0,29 & 0,51 \\
\hline $\begin{array}{l}\text { Taux de variation annuel 1990-2014 dû au solde } \\
\text { migratoire }\end{array}$ & $-0,32$ & 0,48 & 0,87 & 0,13 \\
\hline Part de l'emploi agricole en 2014 (\%) & 7,2 & 2,7 & 8,4 & 2,7 \\
\hline
\end{tabular}

*: communes concernées par l'impluvium

Source : Insee, Recensement de population, 1990, 2014

Tableau 3. Occupation des sols

\begin{tabular}{|l|l|l|l|l|l|l|l|l|}
\hline & \multicolumn{2}{l}{ Langres } & \multicolumn{2}{l|}{ Volvic ${ }^{*}$} & \multicolumn{2}{l|}{ Cévennes } & \multicolumn{2}{l|}{ France } \\
\hline & $\begin{array}{c}1990 \\
(\%)\end{array}$ & $\begin{array}{c}2012 \\
(\%)\end{array}$ & $\begin{array}{c}1990 \\
(\%)\end{array}$ & $\begin{array}{c}2012 \\
(\%)\end{array}$ & $\begin{array}{c}1990 \\
(\%)\end{array}$ & $\begin{array}{c}2012 \\
(\%)\end{array}$ & $\begin{array}{c}1990 \\
(\%)\end{array}$ & $\begin{array}{c}2012 \\
(\%)\end{array}$ \\
\hline Territoires artificialisés & 2,1 & 2,3 & 4,1 & 5,5 & 0,8 & 0,9 & 4,6 & 5,5 \\
\hline Territoires agricoles & 63,5 & 63,5 & 32,0 & 33,4 & 9,2 & 10,1 & 60,1 & 59,5 \\
\hline Terres arables & 25,1 & 25,8 & 0,0 & 0,6 & 1,3 & 1,0 & 28,0 & 28,1 \\
\hline dont cultures permanentes & 0,1 & 0,1 & 0,0 & 0,0 & 0,3 & 0,2 & 2,6 & 2,4 \\
\hline dont prairies & 34,8 & 33,4 & 21,1 & 20,1 & 2,6 & 2,9 & 16,0 & 15,6 \\
\hline dont zones agricoles hétérogènes & 3,5 & 4,2 & 10,9 & 12,7 & 5,0 & 6,0 & 13,5 & 13,4 \\
\hline Forêts & 33,6 & 33,6 & 54,7 & 56,2 & 57,6 & 57,8 & 26,4 & 25,8 \\
\hline Milieux semi-naturels & 0,5 & 0,3 & 9,2 & 4,9 & 32,3 & 31,1 & 7,9 & 8,2 \\
\hline Milieux à végétation arbustive ou herbacée & 0,5 & 0,3 & 9,2 & 4,9 & 30,6 & 29,4 & 6,1 & 6,5 \\
\hline Espaces ouverts, sans ou avec peu de végétation & 0,0 & 0,0 & 0,0 & 0,0 & 1,7 & 1,7 & 1,7 & 1,7 \\
\hline Zones humides, surfaces en eau & 0,3 & 0,3 & 0,0 & 0,0 & 0,1 & 0,1 & 1,0 & 1,0 \\
\hline
\end{tabular}




\begin{tabular}{|l|l|l|l|l|}
\hline Surface totale $\left(\mathrm{km}^{2}\right)$ & 2275 & 38 & 3764 & 548508 \\
\hline
\end{tabular}

* : impluvium (échelle infra-communale)

Source : ministère de la Transition écologique et solidaire, Corine Land Cover, 1990, 2012

\subsection{Analyse des diagrammes des systèmes socio-écologiques des trois territoires} agents de développement, association de protection de l'environnement, conseillers agricoles, forestiers, agriculteurs). La combinaison de zones cultivées et de forêts avec des zones de relief de plateaux et de vallées donne une grande valeur identitaire du paysage pour les habitants qui l'ont intégré dans leurs usages sociaux et pour les touristes qui l'associent au riche patrimoine bâti.

Figure 3. Système socio-écologique du Pays de Langres

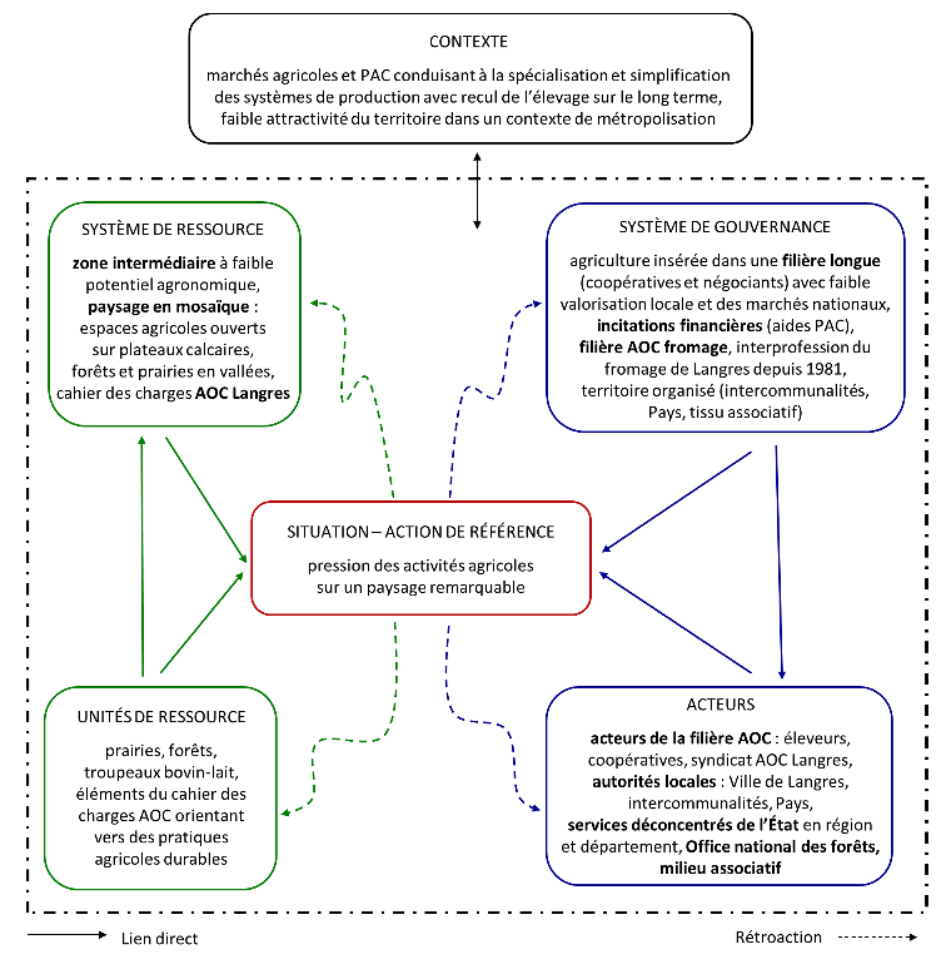

Source : traduit et adapté de Berriet et al. (2016)

Concernant l'identification des facteurs impactant les BSE, le principal frein à la production des BSE est la logique d'agrandissement des exploitations agricoles : avec une surface moyenne de 140 ha, les 786 exploitations de la zone sont dans une logique d'accroissement des superficies de céréales et oléagineux $(+22 \%$ de la surface agricole 
utile entre 2006 et 2014) et une réduction des autres cultures (-72\%), traduisant une spécialisation et simplification des systèmes de production. Ce processus est entretenu par les incitations économiques de marché (prix des céréales, crise du lait et de la viande), par des évolutions technologiques (mécanisation, numérique...), mais aussi par des évolutions dans les représentations du métier d'agriculteur. Les politiques publiques et en premier lieu la PAC tendent à accompagner ce mouvement, notamment par des régimes d'aides plus favorables aux céréales qu'à l'élevage. Rappelons que la PAC comprend les paiements directs (" premier pilier » dont relèvent ces aides directes à la production pré-mentionnées) et les aides au développement rural («second pilier »). Les paiements directs sont destinés à subventionner les zones productives, tandis que les autres paiements du second pilier (tels que les aides aux zones défavorisées ou les mesures agro-environnementales) sont censés subventionner des zones spécifiques moins ou non productives jouant un rôle dans la multifonctionnalité de l'agriculture, telle que la biodiversité ou la gestion du paysage. En l'occurrence, dans le Pays de Langres, le montant moyen d'aides du premier pilier par exploitation est le double de la moyenne nationale et, pour plus de trois quarts d'entre elles, les aides sont découplées. Leur diminution estimée à 20-30\% depuis 2014 par rapport à la période antérieure tend à accentuer les effets relevés ci-dessus. Les conséquences sur les BSE se traduisent sur le territoire de Langres par :

- le déclin de l'élevage et le retournement des prairies ;

- une pression sur la structure du paysage mentionnée par les acteurs rencontrés et illustrée par des photos aériennes : disparition d'arbres isolés, de haies. Cette pression semble s'être accentuée avec l'application de la PAC de 2014 introduisant l'obligation de $5 \%$ de surface agricole d'intérêt écologique ;

- le déclin de la biodiversité observée par l'indice d'abondance d'oiseaux communs qui est en recul significatif depuis 2000 ;

- l'abandon des terres les plus difficiles dans les fonds de vallées notamment.

L'élevage sous signe de qualité (AOC Langres) apparaît comme un facteur positif encourageant la production de BSE. L'activité de transformation fromagère dans cette zone d'élevage a été introduite par une famille suisse dans les années cinquante. Elle s'est progressivement organisée jusqu'à la création du syndicat interprofessionnel du fromage de Langres et sa reconnaissance comme AOC en 1991. Aujourd'hui, la filière qui compte une cinquantaine de salariés regroupe 19 producteurs fabriquant environ 550 tonnes de fromages par an. La reconnaissance de la qualité du produit par le consommateur passe par un cahier des charges strict, notamment sur l'alimentation animale (six mois de mise à l'herbe minimum). L'appellation permet d'améliorer la rentabilité économique des élevages laitiers. Une étude sur les appellations Chaource et Époisses (appellations présentes sur le territoire) aux cahiers des charges proches (Soulignac, 2010) a montré des effets positifs sur la biodiversité (plus grand nombre d'espèces végétales présentes, plus forte présence de haies). Ces effets positifs sont cependant marginaux compte tenu de la faible dimension économique de cette filière.

À Volvic (figure 4), l'enjeu central porte sur la préservation de la qualité et de la disponibilité des eaux souterraines. Le système de ressource peut être décrit comme un système complexe composé du sous-sol (impluvium), le sol, et le paysage reconnu récemment par une inscription au patrimoine mondial de l'Unesco. Ces trois éléments sont interconnectés et forment un seul système qui donne sa valeur à l'eau de Volvic. Ces eaux sont exploitées à la fois par un syndicat, le Syndicat mixte des utilisateurs de 
l'eau de la région de Riom (Smuerr), et par la société Danone, le leader agro-alimentaire français qui a racheté la société des eaux de Volvic en 1992. Sur le plan quantitatif, la balance forêt-agriculture joue un rôle important dans l'équilibre du système, puisque le couvert végétal forestier intercepte une part significative des précipitations, jusqu'à $45 \%$ pour une forêt de résineux. L'évaporation est donc en général plus importante en forêt que pour d'autres types de couvert. Fermond et al. (2013) montrent qu'à l'échelle du bassin versant de Volvic, " un accroissement de $1 \%$ des surfaces boisées se traduirait par une diminution de 0,2\% du débit moyen des sources». Sur le plan qualitatif, le mode d'occupation du sol est un facteur clé. Le plus grand risque provient des changements de pratiques agricoles des éleveurs qui, jusqu'à présent, ont un recours limité à la fertilisation de synthèse et aux produits phytosanitaires sur leurs cultures (environ $10 \%$ des surfaces agricoles) et conservent une gestion extensive de leurs prairies naturelles.

Figure 4. Système socio-écologique de l'impluvium de Volvic

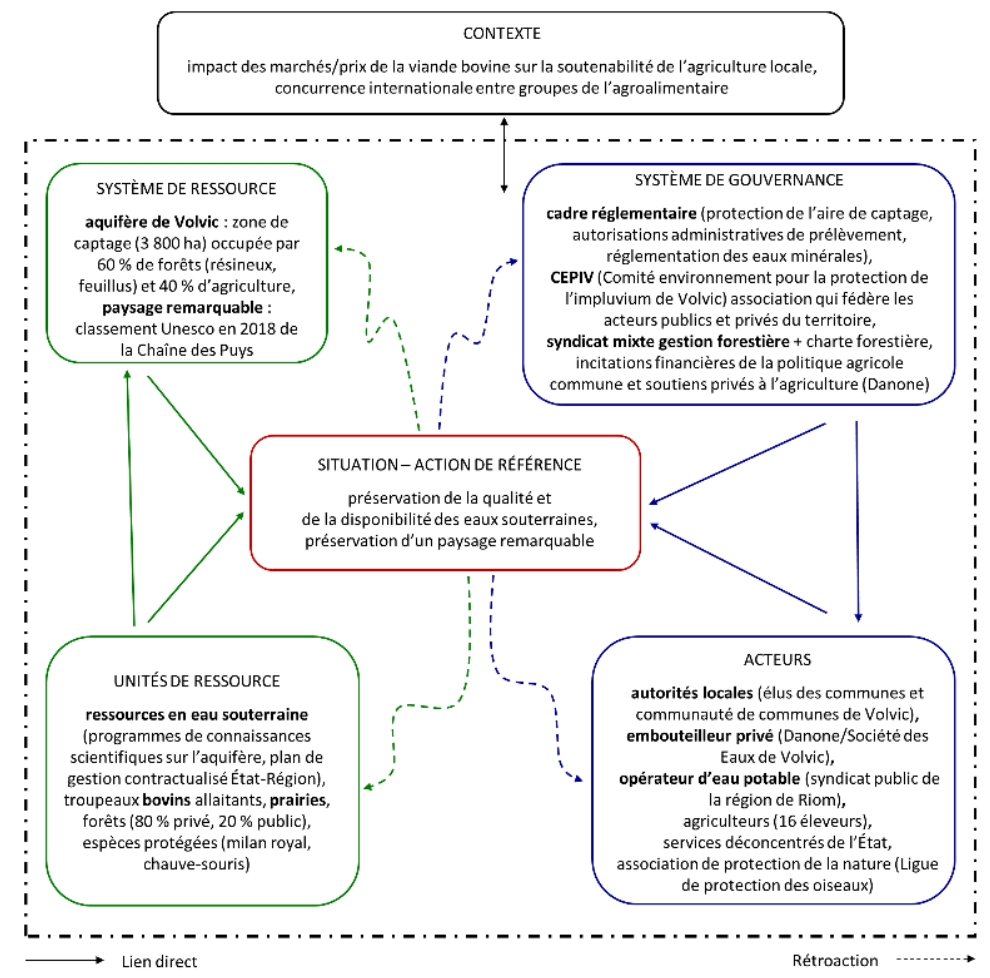

Source : traduit et adapté de Chervier et al. (2016a)

Plusieurs facteurs contribuent à maintenir le système en équilibre et à favoriser cette production de bénéfices environnementaux en matière d'eau et de paysages. Tout d'abord, les incitations économiques à une intensification de l'exploitation de la forêt et des prairies sont limitées. Trop morcelée et relevant de plusieurs centaines de petits propriétaires privés, la forêt est largement sous-exploitée sur le territoire. Quant à l'agriculture, soumise à des prix de marché en baisse (que les aides PAC peinent à contrecarrer), elle est aujourd'hui en fort déclin, avec la disparition des troupeaux laitiers et les élevages bovins allaitants qui se sont généralisés au sein de la quasitotalité des exploitations restantes (plus grandes, mais aussi plus extensives). 

environnement pour la protection de l'impluvium de Volvic) regroupant les quatre communes du bassin versant (élus) et Danone a été créée en 2005. Sa mission est de développer la collaboration dans l'élaboration de politiques appropriées pour la gestion $\mathrm{du}$ bassin versant. À titre d'exemple, les exploitations agricoles bénéficient depuis d'aides à la conversion en agriculture biologique et d'investissements économiques locaux (matériel d'épandage, de stockage) qui sont un facteur important de changement dans les pratiques agricoles. Ces changements sont également plus susceptibles de bénéficier du soutien des agriculteurs s'ils sont économiquement/ techniquement pertinents pour leur exploitation, mais aussi si le processus d'émergence et de diffusion est participatif et garantit la liberté de décision. Des points négatifs existent cependant. En effet, les agriculteurs ne participent pas à la gouvernance du système (ils ne sont pas membres de l'association CEPIV). À terme, cette situation peut générer des dysfonctionnements, en particulier parce que la viabilité économique de l'agriculture dans la zone est très fragile. Les agriculteurs de l'impluvium appellent d'ailleurs Danone à un paiement pour les «services " qu'ils lui rendent gratuitement, rien n'étant ni acté ni formalisé en ce sens actuellement.

Dans le dernier terrain, la situation-action de référence peut être formulée comme suit : alors que le paysage des Cévennes est reconnu par un label international Unesco, certaines stratégies agricoles à court terme contribuent à dégrader ce paysage (figure 5). Dans ce contexte, existe-t-il des actions locales pour contrer une telle évolution? Les Causses et les vallées des Cévennes conservent ainsi de nombreux témoignages de l'évolution sur plusieurs siècles de leurs sociétés pastorales. Leur patrimoine bâti et leurs caractéristiques paysagères qui reflètent le pastoralisme traditionnel sont soutenus par un renouveau contemporain de l'agropastoralisme, notamment dans les vallées cévenoles avec l'arrivée de néo-ruraux. Cette vitalité rurale, qui dans un premier temps peut être considérée comme un BSE en tant que résultante de pratiques agricoles et forestières durables, peut également être définie, dans notre approche systémique, comme un facteur favorable pour expliquer le maintien des paysages agropastoraux. À ce titre, elle participe aux efforts de préservation des paysages agropastoraux pour faire face aux tendances de dégradation de ces paysages: désempierrement, destruction des murets, retournement des prairies... 
Figure 5 : Système socio-écologique du Parc national des Cévennes

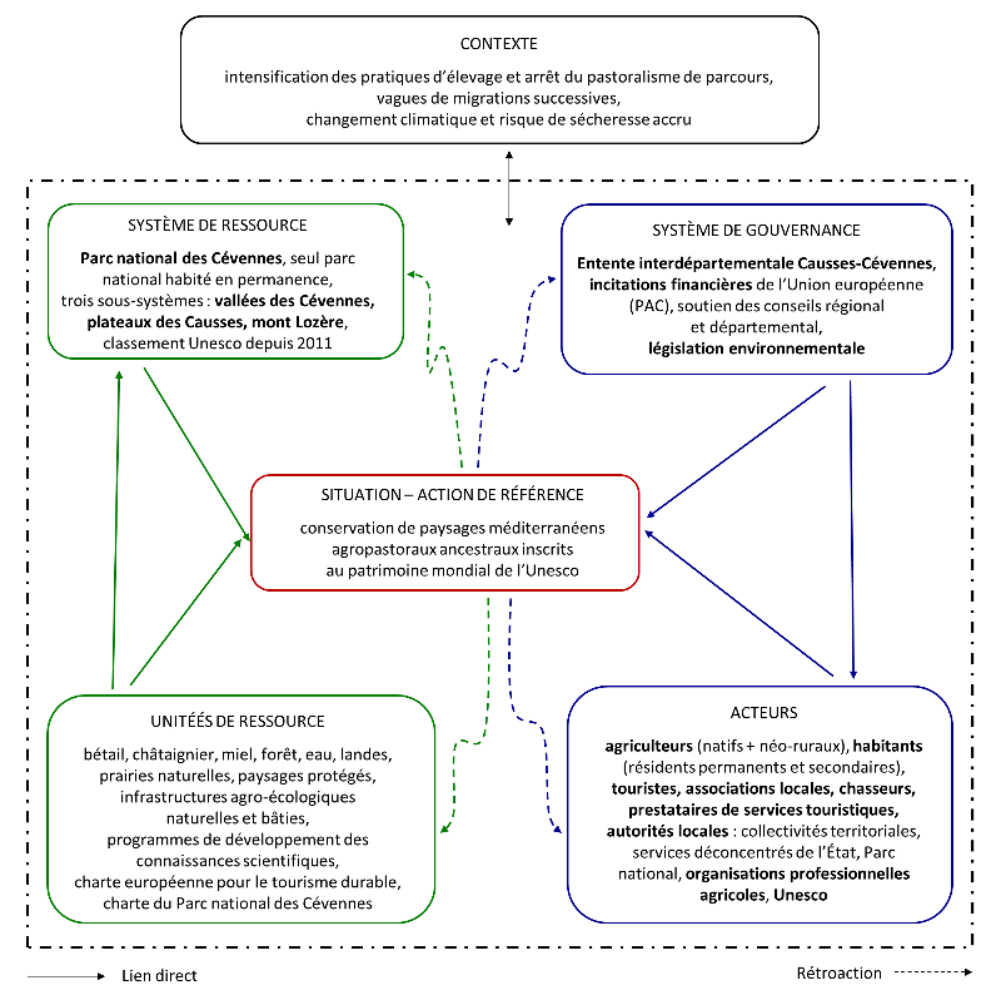

Source : traduit et adapté de Lataste et Piguet (2016)

L'analyse des principaux facteurs influant les BSE, en l'occurrence le paysage agropastoral, pointe le rôle des soutiens financiers de la PAC dans cette zone rurale aux conditions de vie et d'activités difficiles. L'attribution de ces paiements a sensiblement été revue lors de la dernière réforme de la PAC, en particulier les mesures agroenvironnementales que perçoivent la majorité des agriculteurs des Cévennes. Ainsi, depuis 2014, le montant de l'aide directe perçue par l'agriculteur par hectare, pour une parcelle contenant des éléments paysagers ou des infrastructures écologiques (arbres ou haies), dépend du prorata entre la zone productive dans cette parcelle et la zone dite non productive ${ }^{9}$. En raison de ce principe dit de "proratisation» des zones non productives en paiements directs, et des rapports de montant entre les différents types d'aide, la PAC encourage (indirectement) les paysans à homogénéiser leurs parcelles afin d'éviter une perte de subventions directes provoquées par des éléments considérés comme non productifs, tels que murs en pierre ou tas de roches. Cette homogénéisation contribue à simplifier les systèmes agricoles et à détruire des BSE produits par les pratiques agropastorales ancestrales.

Pour contrecarrer l'évolution vers ces pratiques agricoles mettant en péril le paysage agropastoral cévenol, le Parc National et les associations locales jouent un rôle actif en soutenant financièrement les agriculteurs qui s'engagent a contrario dans des démarches agricoles durables. Ainsi, une intervention conjointe du Parc national et du lobby de l'Entente interdépartementale des Causses et Cévennes a conduit le ministère français de l'Agriculture à reconnaître la spécificité locale des pratiques agropastorales en Cévennes, avec une moyenne de 60 \% de zones de châtaigneraies devenues éligibles aux paiements directs dans la zone de l'AOP Pélardon. S'inspirant de cette action, l'association Euromontana et d'autres parties prenantes lancent actuellement une 
pétition pour la reconnaissance du rôle des écosystèmes forestiers dans les activités pastorales.

\subsection{Synthèse des éléments influant sur le développement des bénéfices sociaux et environnementaux} prédominant d'orientation des BSE (prix des produits agricoles et des intrants, soutien à la production par les aides du premier pilier de la PAC...) qui se révèle le plus souvent négatif (dégradation des paysages, pollution et épuisement des nappes phréatiques, atteinte à la biodiversité...). Outre ces deux principaux facteurs, l'article pointe cinq éléments communs identifiés comme facteurs positifs contribuant au développement des BSE.

- Les aides de la PAC dédiées au développement rural (second pilier) contribuent au maintien des activités productrices favorables aux BSE dans nos trois terrains d'étude. Le versement des aides en zones défavorisées (indemnisation compensatoire des handicaps naturels) et des mesures agro-environnementales constituent des sources de revenus non négligeables pour les agriculteurs, conditions nécessaires, mais pas toujours suffisantes, pour promouvoir les pratiques vertueuses au regard des enjeux agro-environnementaux.

- L'importance des processus de régulation apparaît également déterminante, en particulier, au sein des structures de gouvernance existantes. Ces structures n'ont pas forcément vocation à gérer les BSE, mais leur présence assure un cadre de dialogue et d'interaction qui concourt à des mécanismes de régulation (Parc national, syndicats, Pays...). Dans le cas de Volvic, ce dialogue est géré à deux échelles: locale et départementale. À l'échelle locale (celle de l'impluvium), le dialogue entre acteurs du territoire est organisé, depuis 2005 et à l'initiative de Danone, dans le cadre de l'association CEPIV. À l'échelle départementale, un comité de suivi de l'arrêté préfectoral d'autorisation de prélèvement des eaux de Volvic a été mis en place en 2014. Ce comité réunit les services de l'État (Direction départementale des territoires, Agence régionale de santé, Direction régionale pour l'environnement, l'aménagement et le logement), un bureau d'études (Bureau de recherches géologiques et minières) et les exploitants de l'eau du territoire (Smuerr et Danone). Ce comité est un lieu de régulation des pratiques des deux utilisateurs principaux des sources de Volvic qui ont atteint aujourd'hui la capacité maximale autorisée de prélèvement (167 litres/seconde pour le Smuerr; 80 litres/seconde pour Danone), alors même qu'ils prévoient tous deux d'augmenter leur prélèvement, justifié par l'augmentation de la population pour le premier et par l'évolution du marché pour le second. Étant donné que le volume total de l'impluvium n'est pas explicitement connu aujourd'hui, une rivalité peut émerger entre les deux demandes. Dans les Cévennes, des interrelations se développent de l'échelle internationale à locale (dépôt par l'entente interdépartementale et le Parc d'un dossier à l'Unesco) en lien avec l'échelon européen (adaptation locale des mesures de la PAC). Dans ces deux cas, une diversité de parties prenantes privées et publiques sont impliquées. Dans le Pays de Langres, la mise en place de l'AOC Langres se traduisant par la régulation d'une filière grâce à un cahier des charges exigeant est associée à des pratiques favorables à la préservation de la qualité paysagère.

- L'accès à des connaissances nouvelles joue également un rôle important dans la production de BSE, tant au niveau de la construction du problème initial que dans l'identification de 
solutions pour le résoudre. Ces connaissances croisent performances économiques et modalités agro-techniques destinées à atteindre les objectifs environnementaux et/ou sociaux identifiés. Dans les Cévennes, la reconnaissance de la spécificité des zones de chênaies et de châtaigneraies pour les petits herbivores (productions de référence spécifiques sur ces systèmes agropastoraux) a permis l'assouplissement de certains critères d'octroi d'aides publiques de la PAC avec l'appui du Parc national des Cévennes. C'est ainsi que la zone de production du Pélardon a été retenue comme lieu d'expérimentation des pratiques agropastorales à l'échelle de l'Union européenne afin de penser l'adaptation des aides publiques du second pilier. À Volvic, la production de données agronomiques et hydrogéologiques est essentielle pour mesurer les risques de pollution de la ressource souterraine, enjeu majeur pour les collectivités territoriales et Danone.

- La définition d'une vision prospective et/ou le développement de cadre de référence pour un développement durable des activités semblent également des facteurs importants pour stimuler la production de BSE. La société Danone à Volvic, anticipe ainsi la disponibilité en eau potable de qualité à moyen et long terme en incitant les agriculteurs à des changements de pratiques. L'impulsion initiale a été donnée en 2015 par la volonté de lutter contre les campagnols terrestres, alors qu'un argumentaire reposant sur la diminution de la fertilisation ou l'usage restreint des phytosanitaires n'aurait sans doute pas été suffisant, car trop associé à un risque de perte potentielle de revenus à court terme, la situation économique des éleveurs allaitants du Massif Central étant déjà difficile. L'action de Danone est, certes, dictée par des impératifs commerciaux, mais il est intéressant de noter que cette finalité industrielle rejoint l'intérêt commun des populations, puisque la même ressource souterraine est également exploitée par un syndicat public qui alimente en eau potable environ 65000 habitants de la région. Ainsi, la volonté de préserver la qualité de l'eau se traduit, à court terme, par un soutien financier indirect des initiatives locales (financement de piégeurs, participation à une coopérative d'utilisation de matériel agricole locale...) afin d'obtenir une confiance des agriculteurs indispensable pour mener des négociations. Mais il s'agit, dans ce cas, de conjuguer court terme et long terme, intérêts publics et privés. Dans les Cévennes, la signature de la charte européenne de tourisme durable, notamment grâce à l'association Cévennes Écotourisme, illustre également cette volonté de mettre en place des cadres d'une action collective durable. Enfin, dans le Pays de Langres, dans un contexte de recul de l'élevage, la capacité des acteurs locaux à faire reconnaître en AOC le fromage de Langres, appuyé sur un cahier des charges relativement exigeant, donne des perspectives à cette filière à la fois au plan économique (volume en croissance) et au plan environnemental en favorisant la production de BSE. Par ailleurs, le rôle structurant du Pays dans la définition de la stratégie de développement du territoire se traduit par un soutien ancien et pérenne à de multiples initiatives agricoles (installations, circuits courts...), permettant à des exploitations de construire des voies de développement agricole alternatives à la spécialisation et à l'agrandissement.

- Enfin, le rôle essentiel du capital social local et de l'impulsion du monde associatif se retrouve en particulier dans deux des trois études de cas (Cévennes et Langres), et de manière moindre à Volvic où seule l'action de la LPO en partenariat avec l'entreprise Danone pour protéger le milan royal (prédateur du campagnol) peut être notifiée. L'innovation pour développer des pratiques innovantes est animée, par exemple, par les néo-ruraux des vallées cévenoles: ouverture de magasins collectifs de vente directe, création d'un atelier de transformation à Florac et d'un centre de formation pour adultes où les producteurs peuvent créer de nouveaux produits transformés. S'il est difficile de quantifier les bénéfices associés à la 
création de ces nouveaux produits, ces innovations organisationnelles n'en structurent pas moins le tissu économique des acteurs locaux.

\section{Conclusion : quels enseignements en termes d'action collective dans les espaces ruraux?} tirer directement utilité dans une relation marchande classique. Ils ne sont pas tous visibles avant qu'il y ait une «mise en consensus» au niveau du territoire pour leur reconnaissance. Oubliée dans la théorie économique standard, la coconstruction de « vision » entre acteurs s'avère une étape indispensable dans la reconnaissance des BSE. Le cadre d'analyse SSE permet dès lors une mise en lumière de l'importance des mécanismes de gouvernance entre les divers acteurs porteurs de cette vision commune, et ce, à différentes échelles d'intervention. Dans les cas étudiés, les acteurs ont su également mobiliser des ressources supplémentaires extérieures, pour produire (cas de Volvic) ou pour maintenir les BSE qu'ils estiment importants à protéger (le paysage en mosaïque à Langres, les pratiques agropastorales des Cévennes). La nature des ressources extérieures est multiple: aides PAC, inscription au patrimoine Unesco, projets de recherche, etc. Les dispositifs d'intervention publique y jouent un rôle important, soit en tant que défenseur de valeur historique, soit en tant que fédérateur et garant de processus de gouvernance. Le cadre d'analyse SSE permet ainsi de mieux appréhender les mécanismes sous-tendus
par le système de ressource, porteur des bénéfices sociaux et environnementaux. En Si cette intervention des acteurs publics peut influer sur la «révélation» des ressources (cas des Cévennes) en étant plus animateurs que régulateurs ou entrepreneurs (Cooke et Morgan, 1998), les acteurs privés participent aussi de ce processus collectif (cas de Volvic, associations cévenoles, filière fromage de Langres) dans des jeux d'interrelations public-privé. 

territoriale, il existe une adaptation des dispositifs proposés par les pouvoirs publics au contexte local. Le cas des Cévennes et de la prise en compte des spécificités de l'agropastoralisme dans le calcul des aides de la PAC illustre bien cette transposition locale revendiquée par une entente interdépartementale soutenue par le Parc national. Les études sur les impacts environnementaux du cahier des charges de l'AOC de Langres donnent également des éléments tangibles de ces effets positifs, même s'ils ne concernent qu'une très faible part de l'agriculture du territoire.

In fine, ces trois études de cas permettent d'esquisser quelques évolutions des formes d'intervention des acteurs publics à l'échelle d'un territoire qui pourraient être favorables à la production de BSE :

- une territorialisation/adaptation locale accrue des mesures d'accompagnement, quelle quesoit l'échelle d'intervention publique ;

- un élargissement des bénéficiaires de ces mesures : pas uniquement les agriculteurs, mais aussi les propriétaires fonciers, les forestiers, les syndicats mixtes, les collectivités locales, les associations, etc. ;

- une reconsidération des cofinancements publics, mais aussi privés, des dispositifs.

Pour le dire autrement, le fait de prendre davantage conscience des phénomènes d'ambiguïté causale (en particulier les coordinations spécifiques et non explicitées) dans la production des BSE suppose d'intégrer dans les conceptions des politiques une nécessaire prise en compte de la dimension historique, mais également la projection dans le futur et la capacité à envisager des effets d'anticipation et la vigueur des projets.

Une telle conception de l'intervention des acteurs publics passerait par un lien étroit avec les acteurs locaux. L'anticipation des trajectoires de développement territorial, en particulier, peut se faire, de façon concertée, au travers par exemple de plans de développement pluriannuels, fondés sur des objectifs et leviers d'action (cas des Plans de développement ruraux européens, déclinés à l'échelle régionale, ou encore des Contrats de plan État-Région français). Dans ces conditions, les politiques publiques territoriales pourraient être garantes de la transmission et de la pérennisation des savoir-faire et pratiques favorables à la production de BSE. Ainsi, elles seraient susceptibles de contribuer à maintenir un patrimoine et des ressources spécifiques régionales, comme c'est le cas de certains conseils régionaux qui ont créé des conservatoires des ressources génétiques sur leur territoire. De telles initiatives peuvent s'avérer fructueuses si elles sont en lien avec la mobilisation de ces ressources par les acteurs. Enfin, l'intervention des acteurs publics à différentes échelles de l'international au local apparait garante du développement économique du territoire. Elle peut empêcher certains effets néfastes, par exemple lorsqu'elle intervient pour éviter la sous-production de facteurs limitants comme dans les systèmes agropastoraux des Cévennes, dont l'équilibre écologique est fragile et où la disparition d'une des composantes (élevage transhumant, existence de pâturages naturels suffisants...) peut déstabiliser tout un système socio-écologique. Pour poursuivre ces recherches dans la même veine d'analyse théorique que celle initiée par Ostrom, il pourrait être intéressant de prolonger notre mise en exergue des BSE par une analyse approfondie des mécanismes et dispositifs qui contribuent à leur valorisation. 


\section{BIBLIOGRAPHIE}

Allaire G., 2013, « Les communs comme infrastructure institutionnelle de l'économie marchande ", Revue de la régulation, vol. 14, https://journals.openedition.org/regulation/10546 ; DOI : https://doi.org/10.4000/regulation.10546.

Allaire G., 2019, « L'ambivalence des communs », Développement durable \& territoires, vol. 10, $\mathrm{n}^{\circ} 1$, https://journals.openedition.org/developpementdurable/13442 ; DOI : https://doi.org/10.4000/ developpementdurable.13442.

Barataud F., Hellec F., Levain A., Petit S., 2018, « Quand les recherches en sciences sociales s'invitent dans la gestion de l'eau ", Natures Sciences Sociétés, vol. 26, n 4, p. 395-406, DOI : https:// doi.org/10.1051/nss/2018054.

Basurto X., Gelcich S., Ostrom E., 2013, « The Social-ecological System Framework as a Knowledge Classificatory System for Benthic Small-Scale Fisheries ", Global Environmental Change, vol. 23, $\mathrm{n}^{\circ} 6$, p. $1366-1380$.

Benko G., Dunford M., Lipiez A., 1996, « Les districts industriels revisités » in Pecqueur B. (éd.), Dynamiques territoriales et mutations économiques, Paris, L’Harmattan, p. 118-134.

Berkes F., Colding F., Folke C., 2002, Navigating Social-Ecological Systems: Building Resilience for Complexity and Change, Cambridge, Cambridge University Press.

Berriet-Solliec M., 2016, Production de biens publics en agriculture : quel accompagnement par la PAC?, Dijon, Educagri Éditions.

Berriet-Solliec M., Lataste F., Lépicier D., Piguet V., 2018, « Environmentally and Socially Beneficial Outcomes produced by agropastoral systems in the Cévennes National Park (France) », Land Use Policy, vol. 78, p. 739-747.

Berriet-Solliec M., Lépicier D., Piguet V., 2016, Landscape and biodiversity in context of strained agricultural and forestry competitiveness, WP 4 Case study Final report FR1 Pays de Langres, Deliverable 4.1, Pegasus H2020 Project $\mathrm{n}^{\circ}$ 633814, London, IEEP, $27 \mathrm{p}$.

Braat L. C., de Groot R., 2012, « The ecosystem services agenda: bridging the worlds of natural science and economics, conservation and development, and public and private policy ", Ecosystem Services, vol. 1, n 1, p. 4-15, DOI : https://doi.org/10.1016/j.ecoser.2012.07.011.

Brondizio E. S., Vogt N. D., Mansur A. V., Anthony E. J., Costa S., Hetrick S., 2016, « A conceptual framework for analyzing deltas as coupled social-ecological systems: an example from the Amazon River Delta », Sustainability Science, vol. 11, n 4, p. 591-609.

Chervier C., Déprès C., Michelin Y., Perrot E., Pham H. V., 2016, Private business and local collaborative watershed management: the case of Volvic in France, WP4 Case study Final Report FR2, Deliverable 4.1, Pegasus H2020 Project $n^{\circ}$ 633814, London, IEEP, 26 p.

Chervier C., Déprés C., Neang M., 2012, « Émergence de la notion de service environnemental et des dispositifs de rémunération des fournisseurs : le cas du Cambodge ", Vertigo, vol. 12, n 3 , https://journals.openedition.org/vertigo/12890; https://doi.org/10.4000/vertigo.12890.

Chervier C., Millet-Amrani S., Méral P., 2016, « Les apports de l'économie institutionnelle à l'analyse des dispositifs de paiements pour services environnementaux : état des lieux et perspectives ", Développement durable \& territoires, vol. 7, n 1, https://journals.openedition.org/ developpementdurable/11280; DOI : https://doi.org/10.4000/developpementdurable.11280. 
Cooke P., Morgan K., 1998, The associational economy: firms, regions and innovation, Oxford, Oxford University Press.

Cooper T., Hart K., Baldock D., 2009, The Provision of Public Goods Through Agriculture in the European Union, Report Prepared for DG Agriculture and Rural Development, Contract $\mathrm{n}^{\circ} 30$ CE-0233091/00-28, London, Institute for European Environmental Policy.

Cox M., 2014, « Applying a Social-Ecological System Framework to the Study of the Taos Valley Irrigation System », Human Ecology, vol. 42, n² 2, p. 311-324.

Dominati E., Patterson M., Mackay A., 2010, « A framework for classifying and quantifying the natural capital and ecosystem services of soils », Ecological Economics, vol. 69, n 9, p. 1858-1868.

Dwyer J., Berriet-Solliec M., Lataste F. G., Short C., Maréchal A., Hart K., 2018, « Social-Ecological Systems as a framework for analysing public benefits from EU farming and forestry, and identifying appropriate policy responses ", EuroChoices, vol. 17, n 3, p. 4-10.

Fermond C., Ferry O., Fiquepron J., Lachassagne P., Rouquet S., 2013, « Principaux enjeux et propositions pour une gestion et une protection harmonieuses des ressources eau et bois ", Revue forestière française, vol. LXV, $\mathrm{n}^{\circ}$ 5, p. 419-444.

Froger G., Méral P., Le Coq J. F., Aznar O., Boisvert V., Caron A., Antona M., 2012, « Regards croisés de l'économie sur les services écosystémiques et environnementaux », Vertigo, vol. 12, n 3, https://journals.openedition.org/vertigo/12900 ; DOI : https://doi.org/10.4000/vertigo.12900.

Howe C., Suich H., Vira B., Mace G. M., 2014, « Creating win-wins from trade-offs? Ecosystem services for human well-being: a meta-analysis of ecosystem service trade-offs and synergies in the real world », Global Environmental Change, vol. 28, p. 263-275.

Knickel K., Maréchal A., 2018, «Stimulating the social and environmental benefits of agriculture and forestry: an EU-based comparative analysis », Land Use policy, vol. 73, p. 320-330.

Lataste F. G., Piguet V., 2016, Conservation of ancestral pastoral landscapes in the Cévennes National Park, WP 4 Case study Final report FR3, Deliverable 4.1, Pegasus H2020 Project $n^{\circ} 633814$, London, IEEP, $36 \mathrm{p}$.

Lataste F. G., Trouvé A., Berriet-Solliec M., Dwyer J., 2015, « La notion de "biens publics" au secours de la Politique agricole commune? », Développement durable \& territoires, vol. 6, $\mathrm{n}^{\circ} 1$, https://journals.openedition.org/developpementdurable/10719; DOI : https://doi.org/10.4000/ developpementdurable.10719.

Marshall A., 1890, Principles of Economics, London, Macmillan.

McGinnis M. D., Ostrom E., 2014, « Social-ecological system framework: initial changes and continuing challenges », Ecology and Society, vol. 19, $\mathrm{n}^{\circ} 2$, article 30.

Méral P., Pesche D. (S/D), 2016, Les services écosystémiques. Repenser les relations nature et société, Versailles, Quæ, $304 \mathrm{p}$.

Ostrom E., 1990, Governing the commons - The Evolution of Institutions for collective action, Cambridge, Cambridge University Press.

Ostrom E., 2005, Understanding Institutional Diversity, Princeton, Princeton University Press.

Ostrom E., 2007, « A diagnostic approach for going beyond panaceas », Proceedings of the National Academy of Sciences, vol. 104, n 39, p. 15181-15187. 
Petit O., 2009, «La politique de gestion des eaux souterraines en France. Une analyse en termes de gestion intégrée », Économie rurale, n 309, p. 50-64, https://journals.openedition.org/ economierurale/300; DOI : https://doi.org/10.4000/economierurale.300.

Soulignac A.-L., 2010, « Ébauche d'état des lieux et indicateurs de suivi du développement durable dans les filières PDO Chaource et Époisses ", mémoire de fin d'étude d'ingénieur agronome AgroSup Dijon, 70 p.

\section{NOTES}

1. Ce projet est un programme de recherche et d'innovation Horizon 2020 financé par l'Union européenne (contrat $n^{\circ} 633814$ ). Le lecteur se reportera au site dédié au programme http://pegasus.ieep.eu/ pour plus de détails (partenaires de la recherche, études de cas, résultats...). Au total, 34 études de cas ont été conduites dans les 10 pays partenaires du programme Pegasus (Knickel et Maréchal, 2018).

2. Par commodité de langage, nous utiliserons le terme BSE tout au long de l'article. À noter que, même si l'adjectif « économique » n'est pas intégré dans le terme BSE, cette notion intègre implicitement les bénéfices économiques directs et indirects des activités agricoles.

3. Mc Ginnis et Ostrom (2014) expliquent qu'au départ, le cadre SSE, envisageait uniquement les "users » de la ressource. Le cadre a ensuite été amendé en intégrant une notion plus large "d'acteurs" qui ne se limite justement pas aux «appropriateurs » des unités de ressource. Dans cet article, nous retiendrons donc le terme plus large d'acteurs.

4. Les rapports détaillés de ces trois études de cas sont accessibles en anglais par le lien http://pegasus.ieep.eu/case-studies/list-of-case-studies\#france.

5. Lors de l'utilisation de données géolocalisées telles que Corine Land Cover, il faut également tenir compte de leur précision dans les interprétations. Ainsi, les données Corine Land Cover utilisées pour caractériser l'occupation des sols ont une résolution spatiale assez grossière (environ 1/100 000) qui ne permet pas d'identifier la nature d'un polygone de moins de 25 hectares.

6. Les zones agricoles intermédiaires sont des zones de production agricole qui ne bénéficient ni de l'important potentiel agronomique permettant des rendements élevés, ni des caractéristiques des zones de montagne bénéficiant à ce titre de soutiens publics de compensation de handicap naturel. Elles sont généralement identifiées comme plus vulnérables sur le plan technique et économique.

7. Le territoire appartient par ailleurs à une autre appellation fromagère, l'Époisses, dont l'aire géographique est plus large, mais qui concerne peu de producteurs dans le Pays de Langres.

8. L'AOP est un signe de reconnaissance européen, alors que le cahier des charges de l'AOC relève d'une procédure française.

9. Par exemple, en France, chaque mètre linéaire de mur de pierre traditionnel équivaut à $1 \mathrm{~m}^{2}$ de surface d'intérêt écologique, chaque mètre linéaire de haie à $10 \mathrm{~m}^{2}$ et un arbre isolé dans une parcelle à $30 \mathrm{~m}^{2}$. 


\section{RÉSUMÉS}

La notion de système socio-écologique d'Ostrom est utilisée ici pour caractériser les pratiques agricoles générant des bénéfices sociaux et environnementaux et identifier les facteurs facilitant ou limitant leur mise en œuvre. Trois études de terrain sont menées en France, relatives aux pratiques agricoles durables en Pays de Langres, à la préservation de la qualité et de la quantité d'eau dans le périmètre de l'impluvium de Volvic et à l'agropastoralisme dans le Parc national des Cévennes. Cinq principaux facteurs sont identifiés qui pointent l'importance de l'intervention publique quand elle relève de formes de gouvernance locale concertée entre acteurs publics et privés à différentes échelles de gestion.

This article uses the Ostrom's notion of socio-ecological system in order to characterize agricultural practices generating social and environmental benefits and to identify factors that ease or limit their implementation. The methodology is based on three case studies in France on sustainable agricultural practices in Langres, about preservation of water quality and quantity in Volvic and on agro-pastoralism in the Cévennes National Park. Five main factors could be identified that point to the importance of public intervention when it matches with concerted local governance between public and private actors at different management scales.

\section{INDEX}

Keywords : governance, common goods, rural development, sustainability, socio-ecological system

Mots-clés : gouvernance, biens communs, développement rural, durabilité, système socioécologique

\section{AUTEURS}

\section{MARIELLE BERRIET-SOLLIEC}

Marielle Berriet-Solliec est professeure d'économie à AgroSup Dijon (UMR Cesaer, AgroSupDijon, Inrae, université Bourgogne Franche-Comté). Responsable en France du programme européen Pegasus, elle s'intéresse à la territorialisation de la politique de développement rural.

\section{CHRISTOPHE DÉPRÉS}

Christophe Déprés est maître de conférences en économie de l'environnement à VetAgro Sup (UMR Territoires, VetAgro Sup). Il travaille sur les déterminants de la fourniture de services environnementaux par l'agriculture.

\section{COLAS CHERVIER}

Colas Chervier est chercheur en économie au Cirad de Montpellier. Il analyse la mise en place de Paiements pour services environnementaux (PSE) dans le domaine forestier.

\section{FRANÇOIS-GAËL LATASTE}

François-Gaël Lataste est ingénieur agronome et docteur en sciences économiques. Il est actuellement chargé d'études et de recherches à BRL Ingénierie. 


\section{DENIS LÉPICIER}

Denis Lépicier est ingénieur de recherche à AgroSup Dijon (UMR Cesaer, AgroSupDijon, Inrae, université Bourgogne Franche-Comté). Il est responsable de la cellule d'études du Cesaer. Ses travaux de recherche et d'expertise portent sur l'évolution des territoires et l'évaluation des politiques.

\section{HAI VU PHAM}

Hai Vu Pham est maître de conférences en analyse des politiques publiques à AgroSup Dijon (UMR Cesaer, AgroSupDijon, Inrae, université Bourgogne Franche-Comté). Il mène des recherches sur les différentes formes de coordination entre acteurs du territoire, au regard des objectifs du développement durable.

\section{VIRGINIE PIGUET}

Virginie Piguet est statisticienne et ingénieure de recherche à INRAE Dijon (UMR Cesaer, AgroSupDijon, Inrae, université Bourgogne Franche-Comté). Elle contribue aux travaux de l'axe « dynamiques et aménagement des territoires » du Cesaer. 